\title{
A Novel Role for Sema3A in Neuroprotection from Injury Mediated by Activated Microglia
}

\author{
Henry H. Majed, ${ }^{1 \star}$ Siddharthan Chandran, ${ }^{1 \star}$ Simone P. Niclou, ${ }^{2}$ Richard S. Nicholas, ${ }^{1}$ Alastair Wilkins, ${ }^{1}$ Mark G. Wing, ${ }^{1}$ \\ Kate E. Rhodes, ${ }^{1}$ Maria Grazia Spillantini, ${ }^{1}$ and Alastair Compston ${ }^{1}$ \\ ${ }^{1}$ Department of Clinical Neurosciences and Centre for Brain Repair, University of Cambridge, Forvie Site, Cambridge CB2 2PY, United Kingdom, and \\ ${ }^{2}$ Graduate School of Neurosciences Amsterdam, Netherlands Institute for Brain Research, 1105 AZ Amsterdam, The Netherlands
}

\begin{abstract}
Microglia exist under physiological conditions in a resting state but become activated after neuronal injury. Recent studies have highlighted the reciprocal role of neurons in controlling both the number and activity of microglia. In this study, microglia derived from newborn rat cortices were cultured and activated by interferon- $\gamma(\operatorname{IFN} \gamma)$ treatment, then exposed to recombinant Sema3A or conditioned medium derived from stressed embryonic cortical neurons. We found that activation of microglia by IFN $\gamma$ induced differential upregulation of the semaphorin receptors Plexin-A1 and Neuropilin-1. This result was confirmed by Northern blotting, reverse transcriptionPCR, and Western blotting. Furthermore, recombinant Sema3A induced apoptosis of microglia when added to the in vitro culture, and a similar result was obtained on activated microglia when Sema3A was produced by stressed neurons. Using an in vivo model of microglia activation by striatal injection of lipopolysaccharide demonstrated a corresponding upregulation of Plexin-A1 and Neuropilin-1 in activated microglia and enhanced production of Sema3A by stressed adult neurons. These results suggest a novel semaphorin-mediated mechanism of neuroprotection whereby stressed neurons can protect themselves from further damage by activated microglia.
\end{abstract}

Key words: microglia; neuron; semaphorin; plexin; neuropilin; neuroprotection

\section{Introduction}

Microglia are resident immune effector cells of the CNS. Under physiological conditions, they exist in resting conditions but become activated in response to neuronal injury. Activation alters both morphological and functional properties of microglia. It is a feature of many inflammatory and degenerative neurological diseases (Kreutzberg, 1996; Benveniste, 1997; McGeer and McGeer, 1999). The phagocytic, antigen-presenting, cytotoxic, and trophic effects of activated microglia are thought to facilitate repair (Elkabes et al., 1996; Polazzi et al., 2001; Harada et al., 2002). Activation has been associated with neuronal death in vitro. It may therefore also contribute to neuronal injury in the context of pathological processes (Chao et al., 1992; Giulian, 1999; Kingham et al., 1999; Liberatore et al., 1999; Streit et al., 1999; Golde et al., 2002). Furthermore, recent studies have highlighted the influence of neuron-derived signaling effects on microglial function, suggesting that controlled elimination of activated microglia may provide a method of limiting the inflammatory response (Neumann and Wekerle, 1998; Liu et al., 2001; Polazzi and Contestabile, 2003).

\footnotetext{
Received May 31, 2004; revised Nov. 11, 2005; accepted Nov. 14, 2005.

This work was supported by a PhD studentship (H.H.M.), a Clinician Scientist Fellowship (S.C.), and a Co-operative Group Grant (A.C. and M.G.S.) from the Medical Research Council, United Kingdom. We thank A. Kolodkin (anti-Npn1), H. Fujisawa (anti-Plexin-A1), and A. Barzilai (anti-Sema3A) for the generous gifts of antibodies.

${ }^{*}$ H.H.M. and S.C. contributed equally to this work.

Correspondence should be addressed to Dr. Alastair Compston, Department of Clinical Neurosciences and Centre for Brain Repair, University of Cambridge, Forvie Site, Robinson Way, Cambridge CB2 2PY, UK. E-mail: alastair.compston@medschl.cam.ac.uk.

DOI:10.1523/JNEUROSCI.0702-05.2006

Copyright $\odot 2006$ Society for Neuroscience $\quad$ 0270-6474/06/261730-09\$15.00/0
}

Semaphorins are a family of secreted and cell-bound signaling molecules defined by the presence of a common 500 aa Sema domain. They are best characterized in relation to axon guidance during development of the nervous system (Tessier-Lavigne and Goodman, 1996; Bagnard et al., 1998; Kolodkin, 1998). Neuropilin and plexin represent the two families of semaphorin receptors that interact to form a coreceptor complex for secreted semaphorins, including Sema3A. Although much remains to be learned about the biological activities and specificities of semaphorins, the functions of Sema3A are mediated primarily through binding to the Neuropilin-1 (Npn-1) and Plexin-A1 coreceptor complex (Takahashi et al., 1999; Tamagnone and Comoglio, 2000). Neuropilins lack a signaling-competent cytoplasmic domain and ensure semaphorin binding, whereas the transmembrane receptor plexin mediates the intracellular response (Kolodkin, 1998; Winberg et al., 1998; Tamagnone et al., 1999).

An emerging body of evidence suggests that semaphorins subserve diverse functions, many unrelated to axon guidance. These include immune modulation, organogenesis, angiogenesis, and cell migration (Behar et al., 1996; Comeau et al., 1998; Gagliardini and Fankhauser, 1999; Miao et al., 1999; Spriggs, 1999; Spassky et al., 2002; Cohen et al., 2003). Sema3A has also been implicated as a mediator of embryonic neuronal apoptosis. Taken with the recent identification of Sema3 A in the adult and injured brain, this suggests a possible role for semaphorin in influencing neural repair (Shirvan et al., 1999; Fujita et al., 2001; Pasterkamp and Verhaagen, 2001). The observation that neurons secrete Sema3A and that Npn-1 and Npn-2 are expressed by activated microglia after middle cerebral artery occlusion suggests 
the potential for reciprocal interaction between neurons and microglia in association with tissue injury (Fujita et al., 2001).

In the present study, we characterized the differential expression profile of neuropilin and plexin in resting and activated microglia. Activated microglia upregulate Npn-1 and Plexin-A1 in vitro and in vivo. We provide evidence that stressed neurons express Sema3A and mediate microglial apoptosis. The death effect of stressed neuron conditioned medium (SNCM) is blocked by inhibition of Npn-1 binding. Together, these findings suggest a novel mechanism whereby neurons exploit the microglial expression of Npn-1 and Plexin-A1, protecting themselves from potentially threatening effects of activated microglia by secreting Sema3A.

\section{Materials and Methods}

Microglial cell culture. Mixed glial cultures were prepared following the protocol of McCarthy and de Vellis (1980). Briefly, the forebrains of newborn rat pups were removed, and the meninges were stripped before mechanical and enzymatic dissociation. The resulting cell suspension was plated onto poly-L-lysine-coated $75 \mathrm{~cm}^{2}$ tissue culture flasks. Culture medium, DMEM supplemented with $10 \%$ fetal calf serum (FCS; Sigma, Poole, UK), was changed at $24 \mathrm{~h}$ and twice weekly thereafter. After $8-10 \mathrm{~d}$, a confluent monolayer of astrocytes was apparent, on top of which oligodendrocyte precursors and a loosely attached layer of phasebright microglia could be seen. Microglia were harvested by shaking for 20 min and were resuspended in 2\% B27 (Invitrogen, Gaithersburg, MD)-supplemented DMEM before plating onto sterile 8-well chamber slides ( $\sim 40,000$ cells per well; Labtek; Fisher Scientific, Loughborough, UK) or plastic 6-well plates ( $\sim 2$ million cells per well; Nunclon, Naperville, IL). Contaminating cells were removed by allowing the cell suspension to adhere to the surface for $10 \mathrm{~min}$ at $37^{\circ} \mathrm{C}$ before washing in two changes of medium. Adherent microglia were incubated for $24 \mathrm{~h}$ to allow them to become "quiescent" before culturing for an additional $24 \mathrm{~h}$ in conditioning medium [insulin-free Sato-supplemented DMEM containing bovine serum albumin $(100 \mu \mathrm{g} / \mathrm{ml})$, transferrin $(100 \mu \mathrm{g} / \mathrm{ml})$, progesterone $(0.06 \mu \mathrm{g} / \mathrm{ml})$, putrescine $(16 \mu \mathrm{g} / \mathrm{ml})$, selenite $(0.04 \mu \mathrm{g} / \mathrm{ml})$, thyroxine $(0.4 \mu \mathrm{g} / \mathrm{ml})$, and triiodothryonine $(0.04 \mu \mathrm{g} / \mathrm{ml})]$ with or without interferon- $\gamma(\mathrm{IFN} \gamma ; 100 \mathrm{U} / \mathrm{ml})$ to produce highly enriched populations of activated or resting microglia.

Neuronal cell culture. Neuronal cell cultures were prepared from the cortices of embryonic day 16 (E16) rat embryos (Wilkins et al., 2001). After enzymatic and mechanical dissociation, cells were plated onto freshly prepared poly-L-lysine-coated $13 \mathrm{~mm}$ glass coverslips $\left(1.5 \times 10^{3}\right.$ neurons per coverslip or $2.5 \times 10^{3}$ neurons per coverslip) or poly-Llysine-coated plastic 6-well plates (2 million cells per well) in 2\% B27supplemented medium or insulin-free Sato-supplemented DMEM.

Preparation of SNCM. Neuronal cultures were prepared in poly-Llysine-coated 6-well plates (2 million cells per well) and cultured for 1 or $5 \mathrm{~d}$ in $2 \%$ B27-supplemented medium. Cells were then washed in DMEM and cultured in conditioning medium (insulin-free Sato) for up to $72 \mathrm{~h}$. After centrifugation at $1000 \mathrm{rpm}$ for $5 \mathrm{~min}$ and passage through a $45 \mu \mathrm{m}$ filter, the resulting supernatant was stored at $-20^{\circ} \mathrm{C}$ and diluted $1: 1$ with fresh medium for experiments, unless stated otherwise.

Sema3A-conditioned medium was generated from 293T cells transfected with chicken Sema3A using myc-his tag and culture in 1\% FCSsupplemented DMEM for $24 \mathrm{~h}$ (Koppel et al., 1997). Sema3A activity of conditioned medium was validated using the standard collapse unit assay on E15 rat dorsal root ganglia explants. At a 1:250 dilution, Sema3Aconditioned medium induced $50 \%$ growth cone collapse after $30 \mathrm{~min}$ (Luo et al., 1993). For experimental procedures, Sema3A-conditioned medium was used at a 1:10 dilution with fresh medium (unless stated otherwise) for $24 \mathrm{~h}$ at $37^{\circ} \mathrm{C}$. Control conditioned medium was derived from green fluorescent protein-transfected 293T cells.

For caspase inhibition experiments, $50 \mu \mathrm{M}$ of the pan-caspase inhibitor Z-VAD-FMK (R \& D Systems, Abingdon, UK) was added to the incubation medium for 24 h. Function-blocking anti-Npn-1 antibody (a gift from A. Kolodkin, Johns Hopkins, Baltimore, MD) was used at 100 $\mu \mathrm{g} / \mathrm{ml}$.
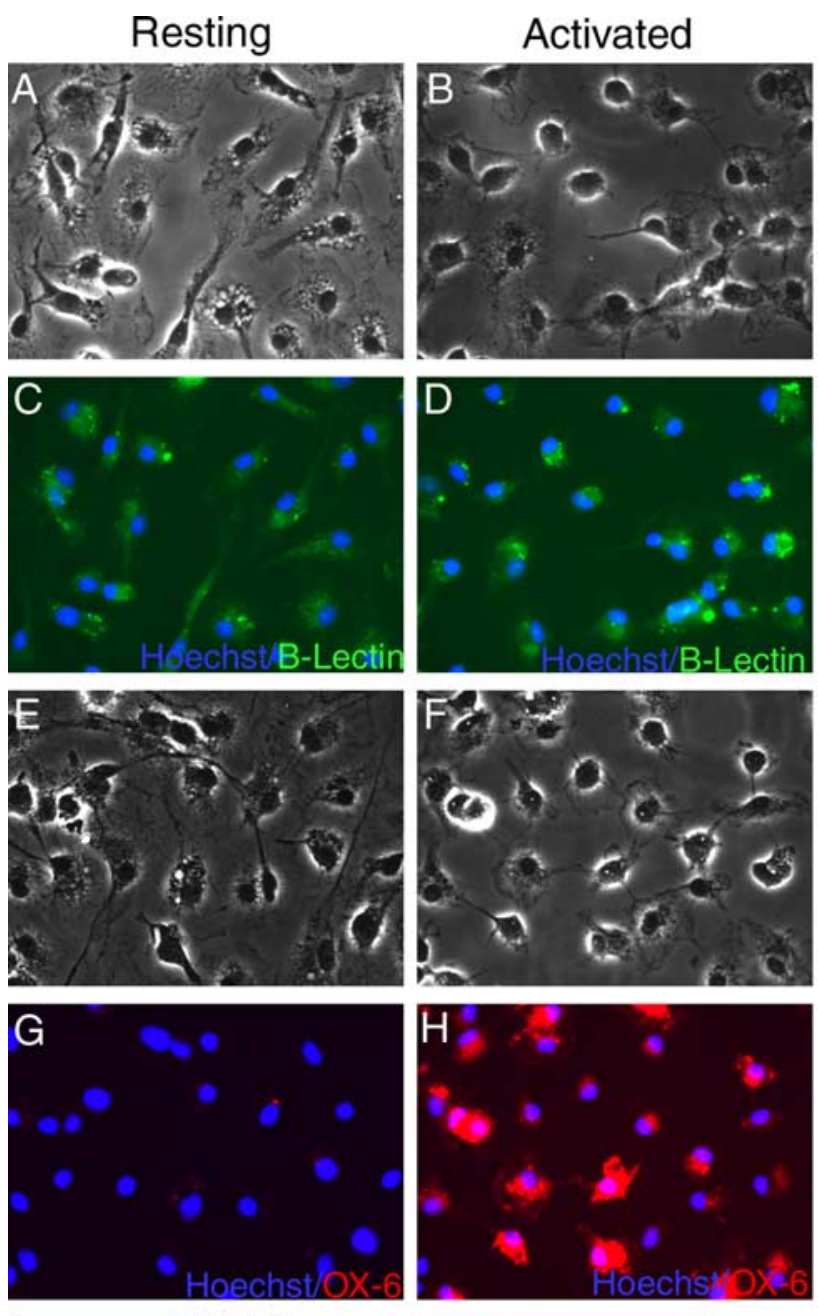

I

$\sim 150 \mathrm{kDa}>$

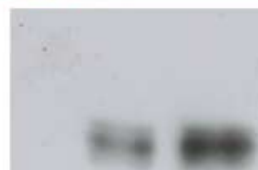

iNOS

$\sim 100 \mathrm{kDa}>$

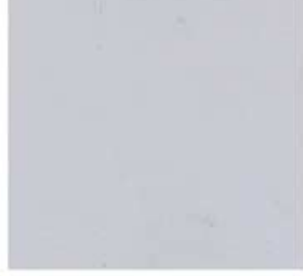

$\sim 50 \mathrm{kDa}>$

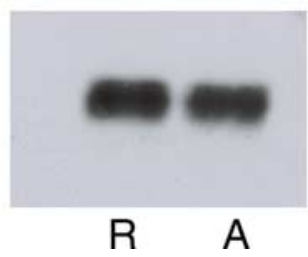

Actin

$\sim 35 \mathrm{kDa}>$

\section{R A}

Figure 1. Characterization of the activation state of microglia. Microglia were purified from mixed glial cultures, plated for $24 \mathrm{~h}$ to become quiescent, and cultured for an additional $24 \mathrm{~h}$ with (right) or without (left) IFN $\gamma$, representing activated and resting microglia, respectively. $A-D$, Representative photomicrographs of microglia under phase-contrast microscopy $(A, B)$ or immunolabeled using an antibody against the microglial marker $\beta$-isolectin $(\boldsymbol{C}, \boldsymbol{D})$ show the distinct morphology of resting $(\boldsymbol{A}, \boldsymbol{C}$ and activated $(\boldsymbol{B}, \boldsymbol{D})$ microglia. $\boldsymbol{E}-\boldsymbol{H}$, Resting microglia are characterized by ramified morphology in contrast to an amoeboid appearance after activation. Immunomicrographs show 0 X6-positive activated microglia after treatment with IFN $\gamma(\mathbf{G}$, $\boldsymbol{H})$ with corresponding phase-contrastimages $(\boldsymbol{E}, \boldsymbol{G}) . \boldsymbol{I}$, Western blot demonstrating upregulation of inducible nitric oxide synthase (iNOS) after IFN $\gamma$-mediated activation of microglia (representative of three independent experiments). Equal loading is confirmed by actin. R, Resting microglia; A, activated microglia. 
Immunocytochemistry. Immunocytochemistry was used to identify cell phenotypes. Cells were stained "live" or after fixation in $4 \%$ paraformaldehyde. The primary antibodies against oligodendrocyte cell-surface markers were galactocerebroside (GalC; 1:4) and A2B5 (1:4) (both derived from hybridoma lines; European Collection of Cell Cultures, Salisbury, UK). Intracellular markers were stained after permeabilization of fixed cultures with $100 \%$ methanol for $10 \mathrm{~min}$ at $-20^{\circ} \mathrm{C}$ : $\beta 4$-isolectin (1:50), OX6 (1:200), and neuronal $\beta 3$-tubulin (1:200) (all from Sigma); and astrocyte GFAP (1:200; Dako, High Wycombe, UK). Flourescenceconjugated secondary antibodies were used to visualize primary antibody staining, and Hoechst 33258 (1:5000 bisbenzamide; Sigma) was added for the final $10 \mathrm{~min}$ of incubation for nuclear identification. Analysis was undertaken using a terminal deoxynucleotidyl transferasemediated biotinylated UTP nick end labeling kit (Boehringer, Bagnolet, France)

Lipopolysaccharide lesion model. Adult rats were killed and perfused with $4 \%$ paraformaldehye $24 \mathrm{~h}$ after basal ganglia microinjection of lipopolysaccharide (LPS; $5 \mu \mathrm{g}$ ) or saline (both in $0.5 \mu$ l volume) using a fine pulled siliconized glass micropipette. After a $1 \mathrm{~h}$ blocking step in $3 \%$ normal goat serum (NGS), $30 \mu \mathrm{m}$ sections were incubated overnight with primary antibody in Tris-buffered saline (TBS) containing $0.2 \%$ Triton X-100 (Sigma) with $1 \%$ NGS. The following antibodies were used: OX42 (1:400; Serotec, Oxford, UK), Plexin-A1 culture supernatant (a gift from H. Fujisawa, National Institute of Genetics, Mishima, Japan) Npn-1

(1:200) (Kolodkin et al., 1997; Pasterkamp et al., 1999), Sema3A (1:250; a gift from A. Barzilai, Tel Aviv University, Tel Aviv, Israel) (Shirvan et al., 2002), and NeuN (1:200; Chemicon, Hampshire, UK). After washing in TBS, sections were incubated in biotinylated secondary antibodies (1:200) for $3 \mathrm{~h}$ at room temperature. After three washes in TBS, sections were incubated in a streptavidin-biotinylated horseradish peroxidase (HRP) complex (ABC kit; Dako) for $2 \mathrm{~h}$ before incubation in chromagen diaminobenzidine $(0.5 \mathrm{mg} / \mathrm{ml}$; BDH Chemicals, Poole, UK) or SG peroxidase substrate (Vector Laboratories, Peterborough, UK) for visualization.

Reverse transcription-PCR. Total RNA was isolated from microglia conditioned for $24 \mathrm{~h}$ with or without IFN $\gamma$, postnatal day 1 (P1) rat cortices, or cortical neuron cultures using Trizol (Invitrogen, Paisley, $\mathrm{UK})$, and poly $\left(\mathrm{A}^{+}\right) \mathrm{RNA}$ was then purified using the Oligotex mRNA mini kit (Qiagen, Hilden, Germany). cDNA was generated by Oligo(dT) primed reverse transcription using the SuperScript II RT-PCR kit (Invitrogen) according to the manufacturer's protocol. Rat Plexin-A1, Plexin-A2, Plexin-A3, Npn-1, and Npn-2 were amplified using primers derived from nucleotide sequences unique for their respective isoforms but conserved between human and mouse plexins and neuropilins (Kameyama et al., 1996a,b; Maestrini et al., 1996). Multiple cDNAs derived from at least three independent amplifications were generated using thermostable Taq polymerase (Promega, Southampton, UK) and sequenced to identify potential errors that may have been introduced during amplification. Primer sequences were as follows, $5^{\prime}$ to $3^{\prime}$ (forward and reverse; all from Sigma Genosys, Haverhill, UK): Plexin-A1, GTGTGTGGATAGCCATCA and CCAGCCTCTCGAACACT; Plexin-A2, TGTGCCCTGCACAACATGT and TGCAGCTCTAGGCCAAACCA; Plexin-A3, GGTGACACCGAACTGGACTT and GACATATTCGGTGGGAATGG; Npn-1, GGCCTCCTGCGATTCGTTACTGCT and CTTAGCCTTGCGCTTGCTGTCATC; Npn-2, TGGATGTACGACCGTGCCAAGTGG and CTGATACTCCATGTCATAG CTGGG; Sema3A, GAAGTTCGACATCATCCTGAGGAC and CTCCATAGACAATTG-
C
Plexin-A1

Plexin-A2

Neuropilin-1

Neuropilin-2

Actin

Plexin-A1

Plexin-A2

Neuropilin-1

Neuropilin-2

Actin $\sim 100 \mathrm{kDa}>$

Plexin-A1 Plexin-A2

$\sim 35 \mathrm{kDa}>$ Actin
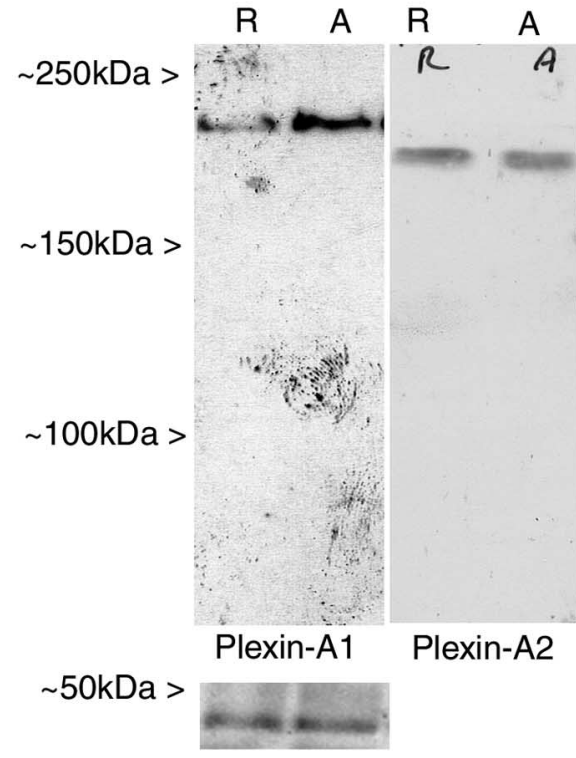

$\sim \sim 35 \mathrm{Ka}>$ Actin 
A

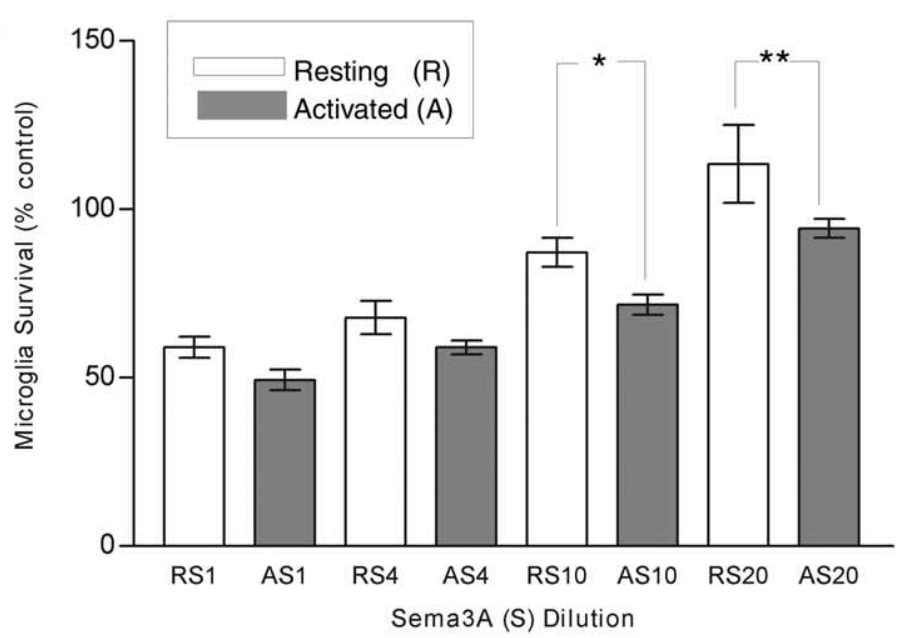

B
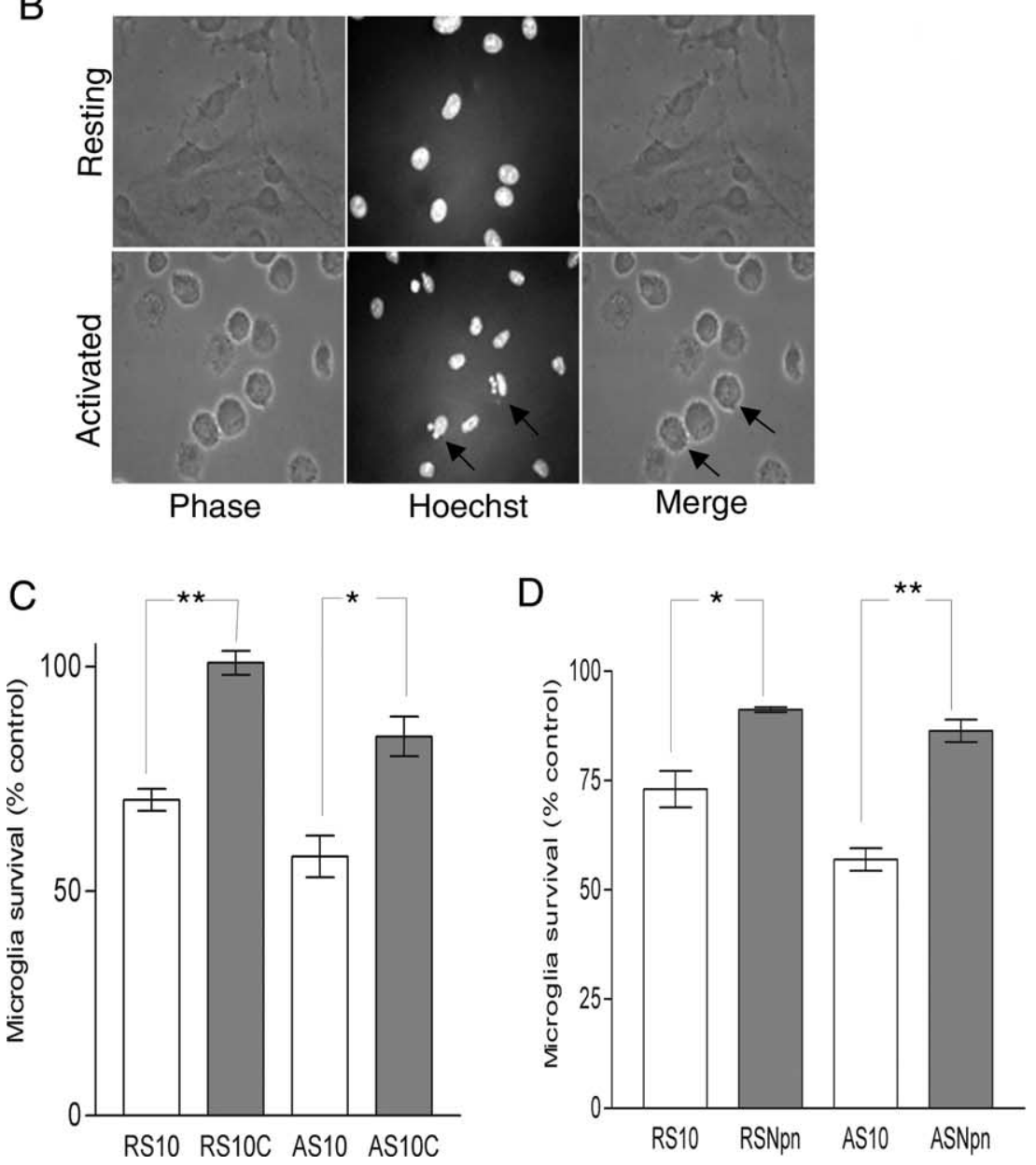

Figure 3. Semaphorin-mediated death of microglia.A, Graph showing the dose-dependent death effect of Sema3A-conditioned medium on microglia, contingent on the activation state. Death is significantly greater in activated $(A)$ microglia than resting $(\mathrm{R})$ cultures at a conditioned medium dilution of 1:10(S10) and 1:20(S20). Cell viability was determined at $24 \mathrm{~h}$ after treatment and is expressed as a percentage with respect to surviving non-Sema3A-treated control cells exposed to an identical amount of $1 \%$ FCS alone. In view of the fact that Sema3A-conditioned medium contains 1\% FCS, appropriate controls were required for each dilution of Sema3A $\left(n=4 ;{ }^{*} p<0.01 ;{ }^{* *} p<0.05\right)$. B, Semaphorin-mediated death is apoptotic. Phase and Hoechstmorphological analysis of nuclei in resting (top row) and activated (bottom row) microglia cultures exposed to a 1:10 dilution of Sema3A-conditioned medium for $24 \mathrm{~h}$. Fragmented and condensed nuclei typical of apoptosis are apparent after Hoechst staining (arrows). C, Inhibition of caspase activity reduces the overall death effect of Sema3A. Significant attenuation of the Sema3A death effect of conditioned medium on resting and activated microglia in the presence (filled bars) compared with the absence (open bars) of pan-caspase inhibitor Z-VAD-FMK $(50 \mu \mathrm{m})$ is observed $\left(n=3 ;{ }^{*} p<0.01 ;{ }^{* *} p<0.001\right)$. D, Function-blocking antibody to Npn-1 inhibits the death effect of Sema3A. Significant attenuation of the Sema3A death effect of conditioned medium on resting and activated microglia in the presence (filled bars) compared with the absence (open bars) of Npn-1 blocking antibody (100 $\mu \mathrm{g} / \mathrm{ml})$ is observed. Microglia were cultured under test conditions for $24 \mathrm{~h}(n=3$; $\left.{ }^{*} p<0.01 ;{ }^{* *} p<0.001\right)$. Error bars indicateSEM. RS, Resting microglia; AS, activated microglia. conjugated secondary antibodies with enhanced chemoluminescence (Renaissance ECL reagent; NEN, Boston, MA).

Cell counting. Cells were viewed using a Leitz microscope. Five consecutive random fields (using a grid) were counted for each coverslip. Results are expressed as mean \pm SEM from at least three different coverslips and at least three separate experiments, unless stated otherwise. Statistical analysis (ANOVA with NewmanKeuls post hoc) was conducted using Prism 2.0 software.

\section{Results}

Activated microglia express Plexin-A1 and Npn-1

Enriched populations of resting and activated microglia from neonatal rat cortex mixed glial cultures were characterized. Microglia (identified by $\beta 4$-isolectinpositive staining) represented $95.5 \pm 1.3 \%$ $(n=3)$ of cells at $24 \mathrm{~h}$ after plating; $3.1 \pm$ $0.5 \%$ were $\mathrm{GFAP}^{+}$astrocytes, and the remainder were $\mathrm{A} 2 \mathrm{~B} 5^{+}$oligodendrocyte precursor cells. Cells were allowed to become quiescent in 2\% B27-supplemented medium for $24 \mathrm{~h}$ before culture for an additional $24 \mathrm{~h}$ with or without IFN $\gamma$ to produce resting or activated microglia. Resting microglia adopted a characteristic ramified morphology compared with the amoeboid appearance of activated microglia, which were OX6 positive with increased inducible nitric oxide synthase expression (Fig. 1).

Initial studies to examine the effect of activation on gene expression in microglia using differential display identified an unknown cDNA, the expression of which was significantly upregulated after activation (data not shown). Homology with corresponding human and mouse sequences identified this transcript as Plexin-A1 (Fig. 2 ). In view of the fact that, together with Npn-1, Plexin-A1 forms part of the highaffinity receptor complex for Sema3A, we next studied the expression profile of other members of the semaphorin receptor family in resting and activated microglia by Northern analysis (Fig. 2A). Upregulation of Npn-1 (and Plexin-A1) after microglial activation was confirmed by reverse transcription-PCR (RT-PCR) and by Western blot analysis (Fig. 2B,C). The other members of the neuropilin-plexin family were not differentially expressed after microglia activation.

\section{Dose-dependent death effect of Sema3A on microglia}

After the demonstration of increased Plexin-A1 and Npn-1 in activated microglia, we next examined the influence of Sema3A on both resting and activated mi- 
croglia. We exposed resting and activated microglia to Sema3A-conditioned medium for $24 \mathrm{~h}$, followed by fixation and immunocytochemistry. Contingent on the activation state, microglia showed a dosedependent death effect, and, in particular, activated cells were more susceptible (Fig. $3 A)$. No effect on survival was observed using control conditioned medium. $\beta$-Lectin and Hoechst counts were comparable, consistent with the purity of the cultures; $>98 \%$ were $\beta$-lectin positive cells at this time point. Interestingly, at a 1:10 and 1:20 dilution, there was a significant differential death effect, and activated microglia were more susceptible to death at each dilution $(p<0.01$ and $p<0.05$, respectively).

Morphological analysis of Hoechstlabeled nuclei and DNA ladder assay (data not shown) demonstrated characteristic fragmented condensed nuclei and DNA cleavage after Sema3A exposure. Moreover, this, together with the finding that the addition of the pan-caspase inhibitor Z-VAD-FMK significantly reduces the death effect of Sema3A after microglia $(p<$ 0.001 ) (Fig. 3C), indicates that Sema3A causes apoptosis of microglia. The addition of anti-Npn-1 function-blocking antibody to microglia cultures significantly inhibited the death effect of Sema3A $(p<0.001)$ (Fig. 3D). Control experiments in nonSema3A-treated cells showed no discernible effect of anti-Npn-1 on microglial survival (data not shown).

\section{Stressed neurons produce Sema3A}

We next examined neuronal expression of semaphorin (Fujita et al., 2001). E16 rat cortical neurons were cultured in 2\% B27supplemented medium for $5 \mathrm{~d}$ and stressed by replacement of medium with insulin-free Sato for up to $3 \mathrm{~d}$. Neuronal cultures were phenotypically characterized at $5 \mathrm{~d}$ in vitro. $\beta$ III-Tubulin ${ }^{+}$ neurons represented $94.6 \pm 1.6 \%(n=3)$ of the cells; the remainder were predominantly astrocytes, with $0.4 \% \mathrm{O}^{+}{ }^{+}$oligodendrocyte precursors and $0.2 \% \mathrm{GalC}^{+}$oligodendrocytes. Immune characterization at $3 \mathrm{~d}$ after supplement replacement revealed $75 \%$ neuronal death. RT-PCR demonstrated Sema3A expression by remaining neurons at 24,48 , and $72 \mathrm{~h}$ after the induction of neuronal stress (Fig. $4 A$ ).

\section{SNCM mediates microglia death}

We next asked whether SNCM could influence microglia survival. SNCM was derived from E16 rat cortical neurons, cultured for $5 \mathrm{~d}$ in 2\% B27-supplemented medium, followed by an additional $3 \mathrm{~d}$ in Sato-only-supplemented medium. Analysis of cultures $24 \mathrm{~h}$ after the addition of SNCM to both resting and activated microglia revealed a significant death effect by SNCM (Fig. $4 B$ ). Activated microglia were significantly more susceptible $(p<0.05)$.

To determine whether death induced by SNCM is attributable to Sema3A present in conditioned medium, we used functionblocking anti-Npn-1 antibody. Anti-Npn-1 ( $\alpha$-Npn-1) resulted in a significant reduction in SNCM-mediated microglial death $(p<0.01)$, suggesting that Sema3A is in part responsible for SNCM-mediated microglial cell death (Fig. 4C). A limited effect was also observed in the resting microglia. This is likely to be
B C
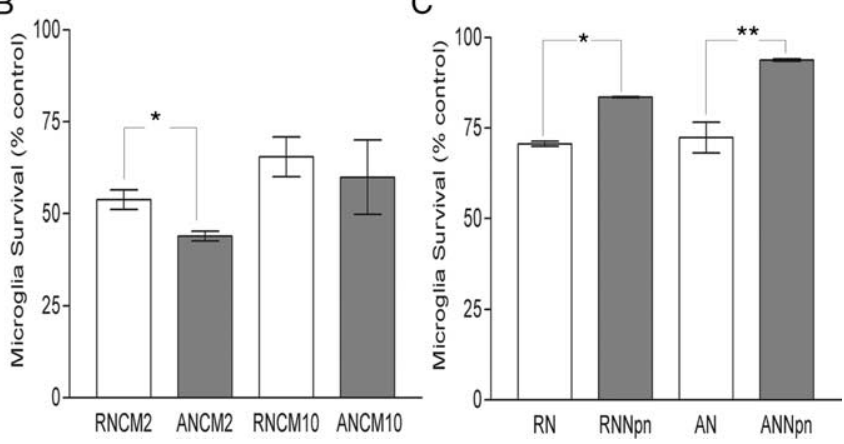
(Div) in 2\% B27-supplemented modium, follow

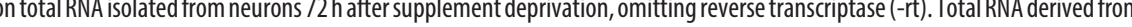
( inhibits the death effect of SNCM when used at a 1:2 dilution, on microglia cultured under test conditions for $24 \mathrm{~h}\left(n=3 ;{ }^{*} p<\right.$ $\left.0.05 ;{ }^{* *} p<0.01\right)$. Error bars indicate SEM.

attributable to the presence of some activated microglia as a result of culturing, even within the resting cultures.

\section{In vivo expression of Sema3A, Plexin-A1, and Npn-1 in adult brain}

To determine whether the identification in vitro of differential Plexin-A1 and Npn-1 expression contingent on the microglia activation state also occurs in vivo, we studied adult rat brains that were killed at $24 \mathrm{~h}$ after the induction of focal injury using LPS. Immunohistological characterization confirmed that LPS injection resulted in an acute inflammatory response characterized by microglial activation (Fig. $5 A-E$ ). Contralateral control lesions of vehicle alone did not produce an acute inflammatory response (Fig. 5A, B,D). Immunohistochemistry for OX42 demonstrated ramified resting microglia in control lesion cortex and amoeboid-appearing activated microglia in LPS lesions. Significantly, Plexin-A1 and Npn-1 immunoreactivity was present only in LPS-mediated inflammatory lesions, colocalized with activated microglia (Fig. $5 \mathrm{~F}-$ $I)$. Sema3A expression was upregulated in the LPS-treated region compared with control lesions (Fig. 6A-D). Furthermore, Sema3A expression was evident in NeuN-positive neurons (Fig. $6 E-G$ ). Western blot analysis of brain sections after LPS or vehicle lesions confirmed significantly enhanced Sema3A expression in LPS-treated tissue (Fig. $6 \mathrm{H}, \mathrm{I}$ ). Consistent with our in vitro findings, in vivo nuclear morphological analysis provides evidence that Sema3A-mediated microglial death is apoptotic (Fig. 7).

\section{Discussion}

Here, we show for the first time that Sema3A can mediate neuronal-microglia interactions. We demonstrate that, contingent on the activation state, microglia differentially express the semaphorin receptors Plexin-Al and Npn-1 in vitro and in vivo. We confirm neuronal expression of Sema3A in vitro and in vivo and provide evidence that stressed neurons are able to mediate microglial death by production of Sema3A. 

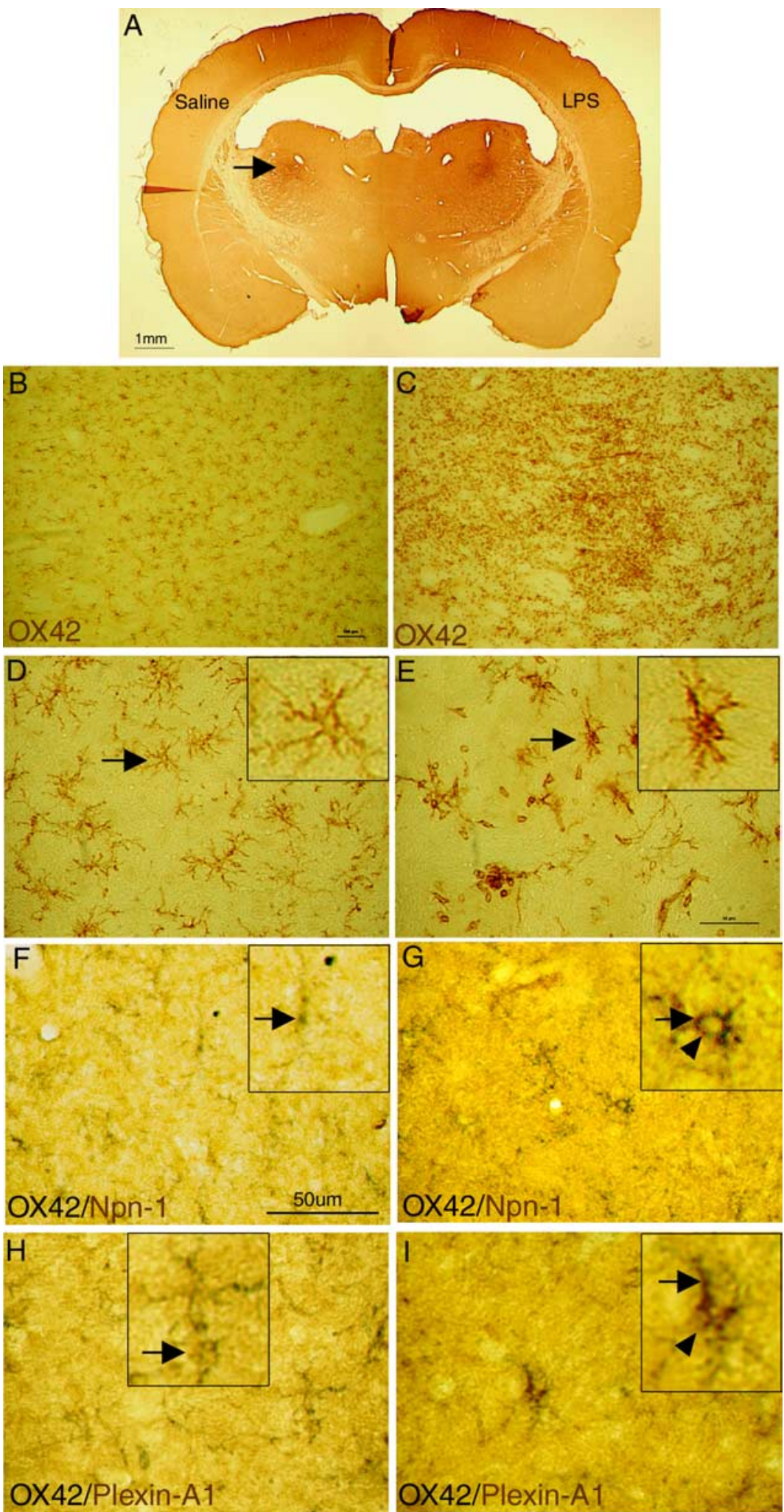

Figure 5. In vivo expression of Plexin-A1 and Npn-1 by microglia. Immunohistochemical analysis of rat brain 24 h after vehicle (left) compared with LPS (right) injections is shown. $\boldsymbol{A}-\boldsymbol{C}$, Representative low-magnification overview of staining with 0X42 showing widespread activation in LPS lesion hemisphere compared with the small area of activation, confined to the injection site, in the control saline hemisphere $(\boldsymbol{A}$, arrow). $\boldsymbol{D}, \boldsymbol{E}$, Higher magnification of $\boldsymbol{B}$ and $\boldsymbol{C}$ detailing increased numbers of $0 \times 42^{+}$-reactive microglia $(\boldsymbol{E}$, arrow), characterized by hypertrophied cell bodies with distinct morphology and heavy accumulation of staining. Resting microglia with a typical ramified appearance ( $\boldsymbol{D}$, arrow) are present in the control lesion hemisphere. $\boldsymbol{F}-\boldsymbol{I}$, Double-labeled immunohistochemistry using 0X42 and Plexin-A1 or Npn-1 antibodies. Neither Plexin-A1 nor Npn- 1 is detected on microglia in the control lesion $(\boldsymbol{F}, \boldsymbol{H})$. After LPS injection, Npn-1-and Plexin-1-positive staining is present in activated microglia $(\mathbf{G}, \mathbf{I})$. Microglia are shown in black (insets, arrows). Brown (diaminobenzidine) identifies Plexin-A1 (G, inset, arrowhead) or Npn-1 (I, inset, arrowhead). Negative controls, omitting the primary antibody, showed no staining (data not shown).
Differential expression of Plexin-A1 and Npn-1 by microglia

The profile of neuropilin and Sema3A has been analyzed in a cerebral model of ischemia, demonstrating microglial expression of Npn-1 and Npn-2 (Fujita et al., 2001). Our findings confirm and extend these observations, highlighting the differential expression profile of Plexin-A1 and Npn-1 between resting and activated microglia. Importantly, the other members of the plexin-neuropilin family were not found to be differentially expressed after microglia activation. We provide evidence in vitro and in vivo for upregulation of both Plexin-A1 and Npn-1 after activation of microglia. Recent reports also implicate semaphorin-mediated neuronal and neuronal progenitor apoptotic cell death (Gagliardini and Fankhauser, 1999; Shirvan et al., 1999, 2002; Bagnard et al., 2001). We therefore examined whether semaphorin has a similar effect on microglia. Exposure to Sema3A-conditioned medium resulted in dose-dependent death of microglia, with activated microglia proving significantly more vulnerable. Death was apoptotic as determined by nuclear morphology, caspase inhibition, and DNA ladder assay analysis. The addition of anti-Npn-1 antibody significantly attenuated the death effect, consistent with the conclusion that Sema3A can mediate apoptosis of microglia.

\section{Microglia-neuron interaction: a role for semaphorin}

An emerging concept is the idea of cross talk between microglia and neurons, an interaction that can be regarded as harmful or beneficial depending on context. In vitro studies demonstrate microglia neurotoxicity through various mechanisms including nitric oxide and NMDAmediated toxicity (Piani et al., 1991; Chao et al., 1992; Kingham et al., 1999). Activated microglia have been implicated in demyelinating and neurodegenerative diseases (Itagaki et al., 1989; Lucchinetti et al., 2000; McGeer and McGeer, 2003). There is some experimental support for the proposal that blocking microglia activation may be neuroprotective, for example through the production of trophic factors including NGF and NT3 (Mallat et al., 1989; Thanos et al., 1993; Elkabes et al., 1996; Rabchevsky and Streit, 1997; Lim et al., 2000; Wu et al., 2002; Zhang et al., 2003). Furthermore, the observation that TNF- $\alpha$ null mice show increased neuronal death after ischemia in association with reduced microglia recruitment is consistent with microglia-mediated neuroprotection 
(Bruce et al., 1996). Studies highlighting the reciprocal relationship between microglia and neurons emphasize the dynamic nature of microglia-neuron interaction (Zietlow et al., 1999; Polazzi and Contestabile, 2003). The precise identity of the protein signals underlying neuron-mediated apoptosis of activated microglia remains unclear (Polazzi and Contestabile, 2003).

Increasing recognition of the importance of semaphorin-mediated signaling in roles other than developmental axon guidance, such as the response to injury, offers a possible biological explanation for the sequence of microglial neuropilin and plexin expression, followed by an apoptotic response to Sema3A. Adult semaphorin expression contributes to the nonpermissive environment of glial scars (Pasterkamp et al., 1999; Niclou et al., 2003). In addition, reciprocal expression in the ischemic adult brain of neuronal Sema3A and microglial neuropilin suggests semaphorinmediated microglia-neuron interaction (Fujita et al., 2001). Our observation that the effect of medium conditioned by stressed neurons on microglia is significantly blocked by anti-neuropilin antibody suggests that neuron-derived Sema3A mediates microglial apoptosis. Additional evidence is provided by the upregulation of neuronal Sema3A, and Plexin-A1 and Npn-1, by activated microglia after LPS-mediated activation in the adult rat brain. Together, these findings indicate that semaphorin is capable of mediating cell death (Shirvan et al., 1999; Bagnard et al., 2001).

Tanaka and Koike (2002) have identified upregulation of a novel microglia gene (mrf-1) after neuronal death that is also implicated in cross talk between microglia and damaged neurons. Our study identifies a novel mechanism of microglia-neuron interaction that may inform the emerging concept of neuronal control of microglia. Because activated microglia are implicated in many disease processes, a biological context for these in vitro and in vivo findings can be readily envisaged (Chao et al., 1992; Giulian, 1999; Kingham et al., 1999; Liberatore et al., 1999; Streit et al., 1999; Neumann, 2001).

In summary, we demonstrate differential expression by activated microglia of the semaphorin receptors neuropilin and plexin. Stressed neurons mediate death of microglia by a semaphorin-dependent mechanism indicating reciprocal regulation of microglia. This may represent a mechanism for containing the inflammatory response to tissue injury whereby the survival of stressed but viable neurons is ensured without compromising the re-
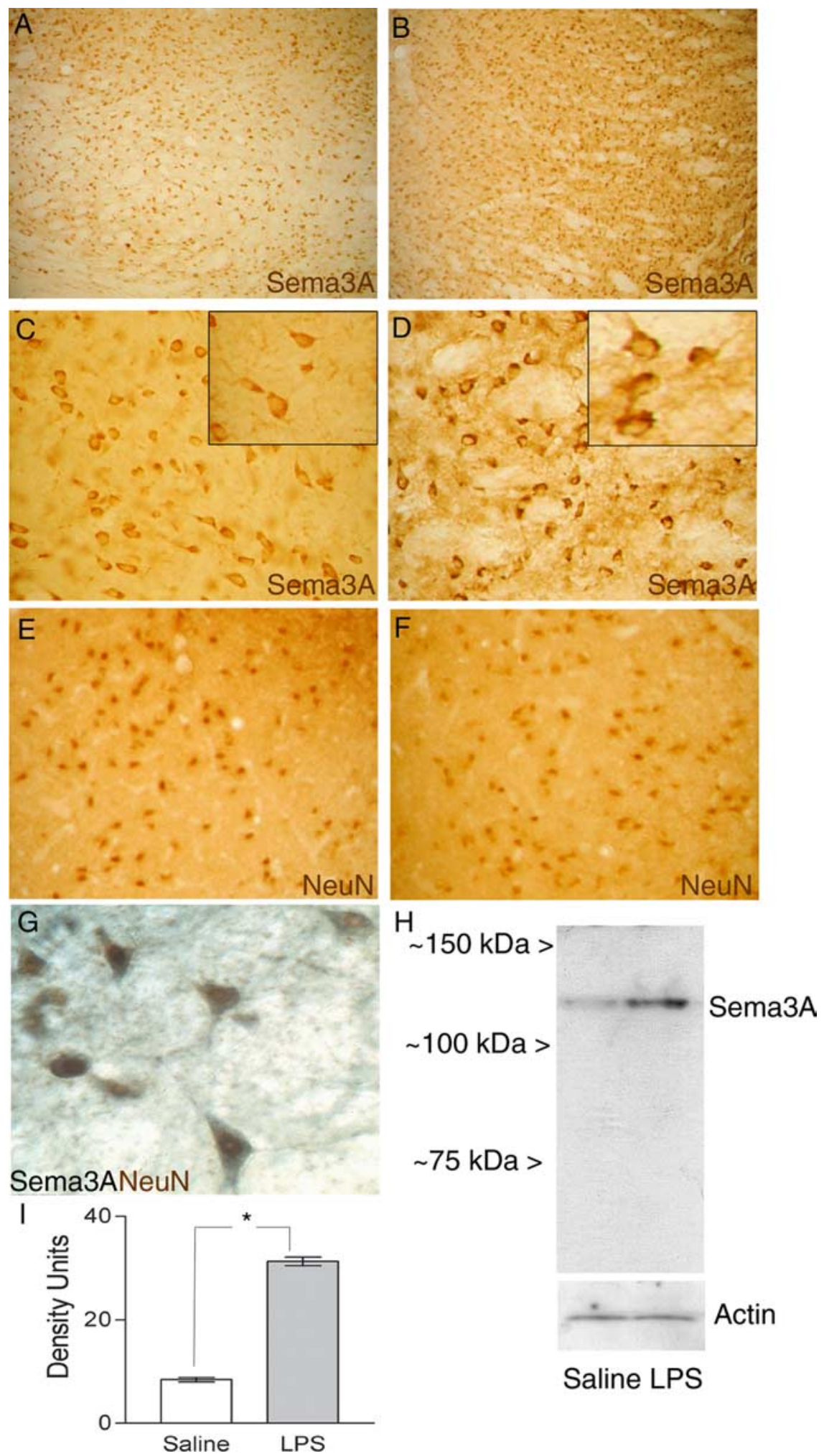

$\sim 100 \mathrm{kDa}>$

$\sim 75 \mathrm{kDa}>$

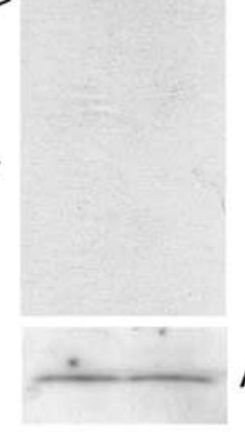

Actin

Saline LPS

Figure 6. In vivo expression of Sema3A by neurons. $A, B$, Immunohistochemical analysis of rat brain 24 hafter vehicle (left) compared with LPS (right) injections. A representative overview of Sema3A staining in cell bodies showing upregulation in both cells and surrounding tissue after $L P S$ lesion $(\boldsymbol{A})$ compared with vehicle injection $(\boldsymbol{B})$ is shown. $\boldsymbol{C}, \boldsymbol{D}$, Higher magnification of $\boldsymbol{A}$ and $\boldsymbol{B}$ showing Sema3A localized to cells with characteristic neuronal morphology. Neurons in the LPS lesion area (D) show marked upregulation of Sema3A compared with vehicle (C).E, $\boldsymbol{F}$, Representative sections immune-labeled using NeuN show distinct nuclear staining in neurons in both vehicle $(\boldsymbol{E})$ and LPS-treated $(\boldsymbol{F})$ sections. Negative controls, omitting the primary antibodies, showed no staining (data not shown). G, Double staining using antibodies against NeuN and Sema3A shows colocalization of NeuN-positive nudei (brown) and Sema3A-positive cytoplasm (blue) after LPS treatment. $\boldsymbol{H}$, Immunoblots of entire hemisphere lysates $24 \mathrm{~h}$ after vehicle (saline) compared with LPS injections show upregulation of Sema3A after LPS lesion. (Equal loading is confirmed using an antibody against actin.) $I$, Relative abundance of Sema3A expression quantified by computerized densitometry of immunoblots $\left({ }^{*} p<0.005\right)$. Results are based on three independent experiments. Errorbars indicate SEM. 


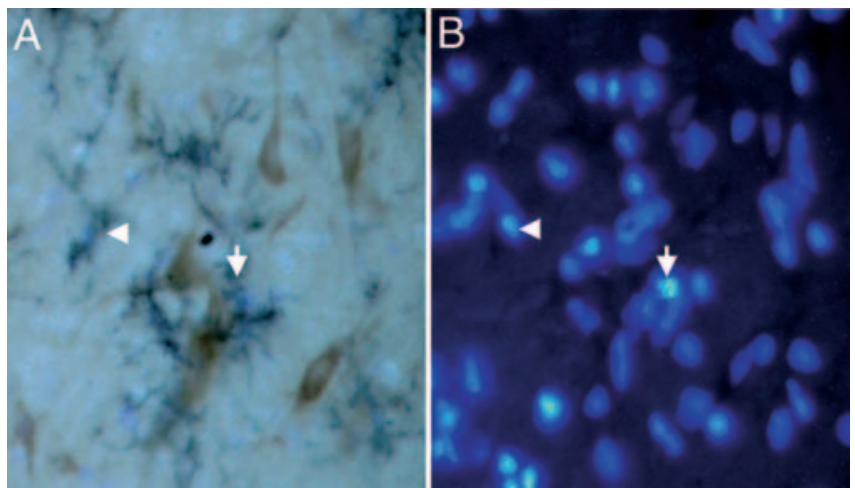

Figure 7. In vivo apoptotic morphology of microglia: spatial localization with Sem3A neurons. Immunohistochemical analysis of rat brain $24 \mathrm{~h}$ after LPS injection. $A, B, A$ representative image showing Sema3A staining in neuronal cell bodies $(\boldsymbol{A}$, brown) and microglia $(\boldsymbol{A}, 0 \times 42$, black) with corresponding Hoescht staining ( $\boldsymbol{B})$. Microglia adjacent to Sema3A-positive neurons have an apoptotic morphology (arrow) in contrast to healthy-appearing microglia in the absence of Sema3A neurons (arrowhead).

moval by microglia of neurons that have already sustained a lethal injury.

\section{References}

Bagnard D, Lohrum M, Uziel D, Puschel AW, Bolz J (1998) Semaphorins act as attractive and repulsive guidance signals during the development of cortical projections. Development 125:5043-5053.

Bagnard D, Vaillant C, Khuth ST, Dufay N, Lohrum M, Puschel AW, Belin MF, Bolz J, Thomasset N (2001) Semaphorin 3A-vascular endothelial growth factor- 165 balance mediates migration and apoptosis of neural progenitor cells by the recruitment of shared receptor. J Neurosci 21:3332-3341.

Behar O, Golden JA, Mashimo H, Schoen FJ, Fishman MC (1996) Semaphorin III is needed for normal patterning and growth of nerves, bones and heart. Nature 383:525-528.

Benveniste EN (1997) Role of macrophages/microglia in multiple sclerosis and experimental allergic encephalomyelitis. J Mol Med 75:165-173.

Bruce AJ, Boling W, Kindy MS, Peschon J, Kraemer PJ, Carpenter MK, Holtsberg FW, Mattson MP (1996) Altered neuronal and microglial responses to excitotoxic and ischemic brain injury in mice lacking TNF receptors. Nat Med 2:788-794.

Chao CC, Hu S, Molitor TW, Shaskan EG, Peterson PK (1992) Activated microglia mediate neuronal cell injury via a nitric oxide mechanism. J Immunol 149:2736-2741.

Cohen RI, Rottkamp DM, Maric D, Barker JL, Hudson LD (2003) A role for semaphorins and neuropilins in oligodendrocyte guidance. J Neurochem 85:1262-1278.

Comeau MR, Johnson R, DuBose RF, Petersen M, Gearing P, VandenBos T, Park L, Farrah T, Buller RM, Cohen JI, Strockbine LD, Rauch C, Spriggs MK (1998) A poxvirus-encoded semaphorin induces cytokine production from monocytes and binds to a novel cellular semaphorin receptor, VESPR. Immunity 8:473-482.

Elkabes S, DiCicco-Bloom EM, Black IB (1996) Brain microglia/macrophages express neurotrophins that selectively regulate microglial proliferation and function. J Neurosci 16:2508-2521.

Fujita H, Zhang B, Sato K, Tanaka J, Sakanaka M (2001) Expressions of neuropilin-1, neuropilin-2 and semaphorin 3A mRNA in the rat brain after middle cerebral artery occlusion. Brain Res 914:1-14.

Gagliardini V, Fankhauser C (1999) Semaphorin III can induce death in sensory neurons. Mol Cell Neurosci 14:301-316.

Giulian D (1999) Microglia and the immune pathology of Alzheimer disease. Am J Hum Genet 65:13-18.

Golde S, Chandran S, Brown GC, Compston A (2002) Different pathways for iNOS-mediated toxicity in vitro dependent on neuronal maturation and NMDA receptor expression. J Neurochem 82:269-282.

Harada T, Harada C, Kohsaka S, Wada E, Yoshida K, Ohno S, Mamada H, Tanaka K, Parada LF, Wada K (2002) Microglia-Muller glia cell interactions control neurotrophic factor production during light-induced retinal degeneration. J Neurosci 22:9228-9236.
Itagaki S, McGeer PL, Akiyama H, Zhu S, Selkoe D (1989) Relationship of microglia and astrocytes to amyloid deposits of Alzheimer disease. J Neuroimmunol 24:173-182.

Kameyama T, Murakami Y, Suto F, Kawakami A, Takagi S, Hirata T, Fujisawa $\mathrm{H}$ (1996a) Identification of plexin family molecules in mice. Biochem Biophys Res Commun 226:396-402.

Kameyama T, Murakami Y, Suto F, Kawakami A, Takagi S, Hirata T, Fujisawa H (1996b) Identification of a neuronal cell surface molecule, plexin, in mice. Biochem Biophys Res Commun 226:524-529.

Kingham PJ, Cuzner ML, Pocock JM (1999) Apoptotic pathways mobilized in microglia and neurones as a consequence of chromogranin A-induced microglial activation. J Neurochem 73:538-547.

Kolodkin AL (1998) Semaphorin-mediated neuronal growth cone guidance. Prog Brain Res 117:115-132.

Koppel AM, Feiner L, Kobayashi H, Raper JA (1997) A 70 amino acid region within the semaphorin domain activates specific cellular response of semaphorin family members. Neuron 19:531-537.

Kreutzberg GW (1996) Microglia: a sensor for pathological events in the CNS. Trends Neurosci 19:312-318.

Liberatore GT, Jackson-Lewis V, Vukosavic S, Mandir AS, Vila M, McAuliffe WG, Dawson VL, Dawson TM, Przedborski S (1999) Inducible nitric oxide synthase stimulates dopaminergic neurodegeneration in the MPTP model of Parkinson disease. Nat Med 5:1403-1409.

Lim GP, Yang F, Chu T, Chen P, Beech W, Teter B, Tran T, Ubeda O, Ashe KH, Frautschy SA, Cole GM (2000) Ibuprofen suppresses plaque pathology and inflammation in a mouse model for Alzheimer's disease. J Neurosci 20:5709-5714.

Liu B, Wang K, Gao HM, Mandavilli B, Wang JY, Hong JS (2001) Molecular consequences of activated microglia in the brain: overactivation induces apoptosis. J Neurochem 77:182-189.

Lucchinetti C, Bruck W, Parisi J, Scheithauer B, Rodriguez M, Lassmann H (2000) Heterogeneity of multiple sclerosis lesions: implications for the pathogenesis of demyelination. Ann Neurol 47:707-717.

Luo Y, Raible D, Raper JA (1993) Collapsin: a protein in brain that induces the collapse and paralysis of neuronal growth cones. Cell 75:217-227.

Maestrini E, Tamagnone L, Longati P, Cremona O, Gulisano M, Bione S, Tamanini F, Neel BG, Toniolo D, Comoglio PM (1996) A family of transmembrane proteins with homology to the MET-hepatocyte growth factor receptor. Proc Natl Acad Sci USA 93:674-678.

Mallat M, Houlgatte R, Brachet P, Prochiantz A (1989) Lipopolysaccharidestimulated rat brain macrophages release NGF in vitro. Dev Biol 133:309-311.

McCarthy KD, de Vellis J (1980) Preparation of separate astroglial and oligodendroglial cell cultures from rat cerebral tissue. J Cell Biol 85:890-902.

McGeer EG, McGeer PL (2003) Inflammatory processes in Alzheimer's disease. Prog Neuropsychopharmacol Biol Psychiatry 27:741-749.

McGeer PL, McGeer EG (1999) Inflammation of the brain in Alzheimer's disease: implications for therapy. J Leukoc Biol 65:409-415.

Miao HQ, Soker S, Feiner L, Alonso JL, Raper JA, Klagsbrun M (1999) Neuropilin-1 mediates collapsin-1/semaphorin III inhibition of endothelial cell motility: functional competition of collapsin-1 and vascular endothelial growth factor-165. J Cell Biol 146:233-242.

Neumann H (2001) Control of glial immune function by neurons. Glia 36:191-199.

Neumann H, Wekerle H (1998) Neuronal control of the immune response in the central nervous system: linking brain immunity to neurodegeneration. J Neuropathol Exp Neurol 57:1-9.

Niclou SP, Franssen EH, Ehlert EM, Taniguchi M, Verhaagen J (2003) Meningeal cell-derived semaphorin $3 \mathrm{~A}$ inhibits neurite outgrowth. Mol Cell Neurosci 24:902-912.

Pasterkamp RJ, Verhaagen J (2001) Emerging roles for semaphorins in neural regeneration. Brain Res Brain Res Rev 35:36-54.

Pasterkamp RJ, Giger RJ, Ruitenberg MJ, Holtmaat AJ, De Wit J, De Winter F, Verhaagen J (1999) Expression of the gene encoding the chemorepellent semaphorin III is induced in the fibroblast component of neural scar tissue formed following injuries of adult but not neonatal CNS. Mol Cell Neurosci 13:143-166.

Piani D, Frei K, Do KQ, Cuenod M, Fontana A (1991) Murine brain macrophages induced NMDA receptor mediated neurotoxicity in vitro by secreting glutamate. Neurosci Lett 133:159-162.

Polazzi E, Contestabile A (2003) Neuron-conditioned media differentially affect the survival of activated or unstimulated microglia: evidence for 
neuronal control on apoptotic elimination of activated microglia. J Neuropathol Exp Neurol 62:351-362.

Polazzi E, Gianni T, Contestabile A (2001) Microglial cells protect cerebellar granule neurons from apoptosis: evidence for reciprocal signaling. Glia 36:271-280

Rabchevsky AG, Streit WJ (1997) Grafting of cultured microglial cells into the lesioned spinal cord of adult rats enhances neurite outgrowth. J Neurosci Res 47:34-48.

Shirvan A, Ziv I, Fleminger G, Shina R, He Z, Brudo I, Melamed E, Barzilai A (1999) Semaphorins as mediators of neuronal apoptosis. J Neurochem 73:961-971.

Shirvan A, Kimron M, Holdengreber V, Ziv I, Ben Shaul Y, Melamed S, Melamed E, Barzilai A, Solomon AS (2002) Anti-semaphorin 3A antibodies rescue retinal ganglion cells from cell death following optic nerve axotomy. J Biol Chem 277:49799-49807.

Spassky N, de Castro F, Le Bras B, Heydon K, Queraud-LeSaux F, BlochGallego E, Chedotal A, Zalc B, Thomas JL (2002) Directional guidance of oligodendroglial migration by class 3 semaphorins and netrin-1. J Neurosci 22:5992-6004.

Spriggs MK (1999) Shared resources between the neural and immune systems: semaphorins join the ranks. Curr Opin Immunol 11:387-391.

Streit WJ, Walter SA, Pennell NA (1999) Reactive microgliosis. Prog Neurobiol 57:563-581.

Takahashi T, Fournier A, Nakamura F, Wang LH, Murakami Y, Kalb RG, Fujisawa H, Strittmatter SM (1999) Plexin-neuropilin-1 complexes form functional semaphorin-3A receptors. Cell 99:59-69.

Tamagnone L, Comoglio PM (2000) Signalling by semaphorin receptors: cell guidance and beyond. Trends Cell Biol 10:377-383.

Tamagnone L, Artigiani S, Chen H, He Z, Ming GI, Song H, Chedotal A,
Winberg ML, Goodman CS, Poo M, Tessier-Lavigne M, Comoglio PM (1999) Plexins are a large family of receptors for transmembrane, secreted, and GPI-anchored semaphorins in vertebrates. Cell 99:71-80.

Tanaka S, Koike T (2002) Selective inflammatory stimulations enhance release of microglial response factor (MRF)-1 from cultured microglia. Glia 40:360-371.

Tessier-Lavigne M, Goodman CS (1996) The molecular biology of axon guidance. Science 274:1123-1133.

Thanos S, Mey J, Wild M (1993) Treatment of the adult retina with microglia-suppressing factors retards axotomy-induced neuronal degradation and enhances axonal regeneration in vivo and in vitro. J Neurosci 13:455-466.

Wilkins A, Chandran S, Compston A (2001) A role for oligodendrocytederived IGF-1 in trophic support of cortical neurons. Glia 36:48-57.

Winberg ML, Noordermeer JN, Tamagnone L, Comoglio PM, Spriggs MK, Tessier-Lavigne M, Goodman CS (1998) Plexin A is a neuronal semaphorin receptor that controls axon guidance. Cell 95:903-916.

Wu DC, Jackson-Lewis V, Vila M, Tieu K, Teismann P, Vadseth C, Choi DK, Ischiropoulos H, Przedborski S (2002) Blockade of microglial activation is neuroprotective in the 1-methyl-4-phenyl-1,2,3,6-tetrahydropyridine mouse model of Parkinson disease. J Neurosci 22:1763-1771.

Zhang SC, Goetz BD, Duncan ID (2003) Suppression of activated microglia promotes survival and function of transplanted oligodendroglial progenitors. Glia 41:191-198.

Zietlow R, Dunnett SB, Fawcett JW (1999) The effect of microglia on embryonic dopaminergic neuronal survival in vitro: diffusible signals from neurons and glia change microglia from neurotoxic to neuroprotective. Eur J Neurosci 11:1657-1667. 